\title{
Auditory Masking Removal for Perception Improvement of Hearing Impaired Persons
}

\author{
B. M. Magdum \\ PG Student, ENTC Dept \\ Sandip Institute of Technology\& \\ Research Center, Nasik, India
}

\author{
P. A. Dhulekar \\ Assistant.Professor,ENTC Dept \\ Sandip Institute of Technology \& Research \\ Center,Nasik,India
}

\begin{abstract}
A novel method is projected in order to get a better speech perception for the hearing impaired people. The algorithm is developed to achieve a specific number of bands of the preferred speech material for dichotic presentation.These bands are obtained using a set of dyadic filters which gives sharp transitions between selected bands. For implementing this algorithm MATLAB software is used .At first by implementing the code eighteen bands of Constant Bandwidth, nineteen bands of $1 / 3$ Octave bandwidths and eighteen bands of critical bandwidth using pair of complementary comb filters from the filter sets are obtained. Ten subjects of various age group participated for the listening test .The test material used for the testing purpose involves a set of 15 Vowel Consonant Vowel (VCV) context .The performed test outcome proves that the projected method is usefull for improving the recognition score and also reduces the response time.
\end{abstract}

\section{Keywords}

Hearing mechanism, Hearing loss, Hearing implants,Dichotic presentation.

\section{INTRODUCTION}

Human auditory system is subdivided into three sections, outer ear(ear cannal) and middle ear, and inner ear (cochlea). The outer ear (ear cannal) and middle ear are responsible for conduction of sound and the inner ear (cochlea) which comprises the main section of human ear called cochlea and the auditory nerves. Hearing disorder is generally categorized according to the location of injury in the auditory system as conductive and sensorineural impairements[1]. Hearing impairment is generally categorized as conductive and sensorineural depending upon the location of damage in the auditory system[1] .Conductive impairment occurs due to injury in the outer ear (ear cannal), ear drum or middle ear,due to which the transmission of sound to the inner ear (cochlea) gets blocked. It generates attenuation of the stimulus and enhance both the Hearing Threshold Levels (HTL) and discomfortable loudness levels. Conductive impairment can be treated clinically or surgically in most cases[1]. When the transduction apparatus of the inner ear (cochlea) gets damaged then such a impairment is known as Sensorineural hearing disorder[1]. The injury caused due to deficiency in the hearing part is known as cochlear disorder and the injury taking place due to defects in the auditory neurons is retrocochlear hearing disorder [1].The cochlear hearing disorder is associated with absence of cochlear hair cells. Impairment caused due to ageing causes malfunctions in hearing mechanism such as stiffening of (BM) i.e basilar membrane, of the cochlea(inner ear) which causes loss of hair cells in the organ of Corti and deterioration of neurons in the intact hearing mechanism. Impairment is bilaterally symmetrical, with greater hearing impairment at high frequencies, and increased difficulty in understanding speech. Initially the hearing disorder can be mild with a loss of sensitivity in the high frequencies that continues to increase with increasing age .When hearing loss occurs due to both conductive and sensorineural loss it is called as mixed hearing loss.Masking effects plays an vital role in our day to day life. When two people are having conversion in silent environment a small quantity of speech power is required for both the person to perceive sound and recognize each other.However, the speech in crowded shopping area is completely impossible to hear. A signal is most likely to get masked by another signal with frequency components which are near to, or the same as, that of the signal. When masking event occurs among any two signals which appear at similar instant of time it is called simultaneous masking or spectral masking, on the other hand, signals which are comparatively postponed in time it is called temporal or non simultaneous masking [10].

\section{PREVIOUS WORK}

Hearing impairment can be moderately remunerated using hearing aid which amplifies acoustic signals with a frequency/gain characteristic which best reimburses for the deficiency in hearing [3]. Conventional hearing aids [1] are typically used for overcoming problem faced due to elevated thresholds.The hearing aids comprises of a microphone, electronic filter, control for the fine-tuning and frequency response, an earphone and a battery for supplying the power source, and elastic tubing and an ear mold, for merging the response of the earphone to the peripheral ear channel. Conventional hearing devices are categorized by their size and the way of wearing, as body-worn hearing aid, eyeglass hearing aid etc[2],[3],[4] .The BTE Hearing devices are the electronic components which are snowed under a tiny oval case that is fitted at backside of the ear. Receiver distributes the responses to the outer ear (ear canal) by means of a flexible tube terminating in an ear mold. These are the hearing aids most commonly used severe to profound hearing impaired. ITE hearing device is made of a small plastic case containing all the components, which is worn in the external section of the peripheral ear and the cochlea. Such aid is normally used by mild to moderate and moderately severe hearing impaired. ITC hearing device is nominal in size, among all hearing aids that fits entirely in the ear canal (outer ear) [1]. Because of small size, available power output is low. Hence mild or moderate, flat, and gradual sloping hearings impaired generally use these aids [2].The compression techniques are used in hearing aids to overcome the problem of acoustic amplification, which compose weak signals audible to intense signals but makes uncomfortably loud due to loudness recruitment in sensorineural hearing impairment. 
In these techniques the amplification reduces with intensity, such that the ample dynamic range of input signal acquired compression in a smaller dynamic range of the output [3]. An epochal method is projected where the critical bands were compacted inorder to regulate the outline of the acoustic filters of hearing impaired persons. The compression using Fourier transform based approach get better value and precision of speech for hearing impaired persons. The compression rates vary between 20 to $40 \%$ depending individual shaped auditory filter [5]. Frequency transposition technique is a method in which energy in the higher frequency region is transposed to lower frequency region.The effects of the simultaneous and non simultaneous masking have been compensated by various speech enhancing schemes in the case of the impairments in the inner ear (cochlea). Some methods which use alteration of energy for certain frequency components are used in order to reduce the spectral masking. Schemes for enhancing the speech perception for the persons with reduced temporal resolution make use of the clear speech [2],[3],[4].

\section{PROPOSED SYSTEM}

Figure 1 represents the block diagram of the proposed system.The blockwise explanation of the system is explained in the following sections.

\subsection{Unprocessed Speech Signal}

The input to the system is the unprocessed speech signals. These are own recorded fifteen VCV signals. These signals are recorded using a recording device.

\subsection{Design of Analysis Filter banks}

Decompose a signal into components of equal or logarithmically decreasing frequency intervals and sample rates. The Dyadic Analysis Filter Bank block decomposes a broadband signal into a collection of successively more bandlimited components by repeatedly dividing the frequency range.

\subsection{Spectral splitting of speech signal}

Spectral splitting of speech signals is done by using a pair of comb filters. The bands of even and odd frequencies are obtained, which are presented dichotically that is odd bands of frequencies are given to the right ear and even bands to the left ear simultaneously.

\subsection{Obtain the desired number of Bands}

In these step the desired number of constant, critical or $1 / 3$ octave bandwidths are obtained here,i.e 18 bandwidths of constant band , 18 bands of critical band and 19 bands of $1 / 3$ octave bandwidths are obtained.

\subsection{Design of Synthesis Filter banks}

The Dyadic Synthesis Filter Bank block typically reconstructs a signal that was decomposed by the Dyadic Analysis Filter Bank block. The reconstruction or synthesis process restores the original signal by upsampling, filtering, and summing the bandlimited inputs in stages corresponding to the analysis process.

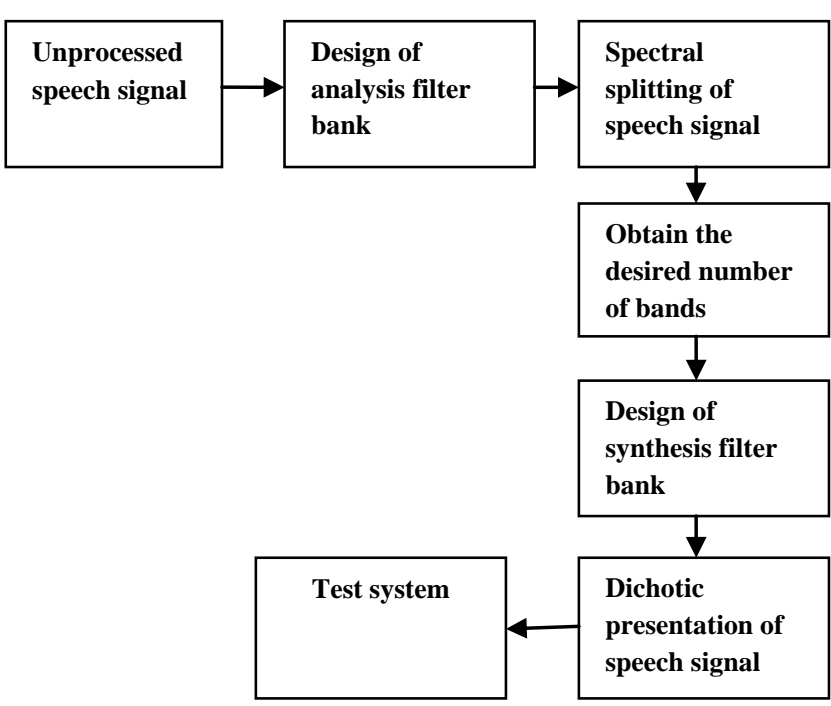

Figure 1: Proposed system

\subsection{Dichotic presentation}

Presenting different signal to both ears is called as dichotic presentation.Dichotic presentation helps in improving the speech perception of hearing impaired persons.

\subsection{Test system}

Test system is used for testing purpose .It contains the unprocessed and processed speech signals which are analyzed and the set of results are obtained.

Table 1 represents the different bands of constant,critical and $1 / 3$ octave bandwidths.

Table 1 : Different Bands

\begin{tabular}{|c|c|c|c|}
\hline $\begin{array}{c}\text { Band } \\
\text { No. }\end{array}$ & $\begin{array}{c}\text { Constant } \\
\text { Bandwidth } \\
\text { Pass band } \\
\text { (KHz) }\end{array}$ & $\begin{array}{c}\text { Critical } \\
\text { Bandwidth } \\
\text { Pass band } \\
\text { (KHz) }\end{array}$ & $\begin{array}{c}\text { 1/3 Octave } \\
\text { Bandwidth } \\
\text { Pass band (KHz) }\end{array}$ \\
\hline 1 & $0.001-0.028$ & $0.01-0.20$ & $0.0708-0.089$ \\
\hline 2 & $0.027-0.056$ & $0.20-0.30$ & $0.089-0.112$ \\
\hline 3 & $0.056-0.084$ & $0.30-0.40$ & $0.112-0.141$ \\
\hline 4 & $0.084-1.111$ & $0.40-0.51$ & $0.141-0.178$ \\
\hline 5 & $1.111-1.396$ & $0.51-0.63$ & $0.178-0.224$ \\
\hline 6 & $1.396-1.167$ & $0.63-0.77$ & $0.224-0.282$ \\
\hline 7 & $1.167-1.950$ & $0.77-0.92$ & $0.282-0.355$ \\
\hline 8 & $1.950-2.227$ & $0.92-1.08$ & $0.355-0.447$ \\
\hline 9 & $2.227-2.505$ & $1.08-1.27$ & $0.447-0.562$ \\
\hline 10 & $2.505-2.782$ & $1.27-1.48$ & $0.562-0.708$ \\
\hline 11 & $2.782-3.059$ & $1.48-1.72$ & $0.708-0.891$ \\
\hline 12 & $3.059-3.336$ & $1.72-2.00$ & $0.891-1.120$ \\
\hline 13 & $3.336-3.613$ & $2.00-2.32$ & $1.120-1.410$ \\
\hline 14 & $3.613-3.891$ & $2.32-2.70$ & $1.410-1.780$ \\
\hline 15 & $3.891-4.168$ & $2.70-3.15$ & $1.780-2.240$ \\
\hline
\end{tabular}




\begin{tabular}{|c|c|c|c|}
\hline 16 & $4.168-4.445$ & $3.15-3.70$ & $2.240-2.820$ \\
\hline 17 & $4.445-4.722$ & $3.70-4.40$ & $2.820-3.550$ \\
\hline 18 & $4.722-5.000$ & $4.40-5.00$ & $3.550-4.470$ \\
\hline 19 & & & $4.470-5.000$ \\
\hline
\end{tabular}

Table 3. Comparision of Recognotion Scores

\begin{tabular}{|c|c|c|c|c|}
\hline Subjects & U .S & $\begin{array}{c}\text { P.S } \\
\text { C.bands }\end{array}$ & $\begin{array}{c}\text { P.S } \\
\text { Critical } \\
\text { bands }\end{array}$ & $\begin{array}{c}\text { P.S 1/3 } \\
\text { Octave } \\
\text { bands }\end{array}$ \\
\hline DMM & 66 & 100 & 80 & 100 \\
\hline PPP & 86 & 100 & 86 & 100 \\
\hline APS & 73 & 100 & 86 & 86 \\
\hline PMN & 73 & 86 & 93 & 93 \\
\hline VRM & 46 & 100 & 100 & 100 \\
\hline RMM & 53 & 80 & 80 & 86 \\
\hline PAP & 46 & 100 & 86 & 100 \\
\hline SPP & 40 & 80 & 100 & 86 \\
\hline NAR & 60 & 86 & 100 & 80 \\
\hline SNR & 40 & 73 & 93 & 100 \\
\hline
\end{tabular}

\section{RESULTS}

Results of listening tests for Response time ,recognition scores and recognition grades are obtained with the 3 set of filter which are are presented in the following sections.

\subsection{Response times}

Table 2 represents the response times for the unprocessed signals(U.S), (P.S) processed signals using constant band (C.bands), critical band(and $1 / 3$ octave bands for the ten subjects DMM (F, 15) PPP(M,13) APS(F,21) PMN(M,26), $\operatorname{VRM}(\mathrm{F}, 31), \operatorname{RMM}(\mathrm{M}, 38)$,PAP(M,51) SPP(F,45) SNR(F,65) NAR(M,70).Figure 2 represents the graphical comparisons of response times.

Table 2 :Comparision of Response Times

\begin{tabular}{|l|l|l|l|l|}
\hline Subjects & U .S & $\begin{array}{l}\text { P.S } \\
\text { C.bands }\end{array}$ & $\begin{array}{l}\text { P.S } \\
\text { Critical } \\
\text { bands }\end{array}$ & $\begin{array}{l}\text { P.S 1/3 } \\
\text { Octave } \\
\text { bands }\end{array}$ \\
\hline DMM & 2.3753 & 1.3057 & 1.2881 & 1.0501 \\
\hline PPP & 1.7220 & 2.1992 & 1.8395 & 1.0300 \\
\hline APS & 1.2251 & 1.0952 & 1.1614 & 1.3754 \\
\hline PMN & 2.4233 & 1.3639 & 1.6055 & 1.5613 \\
\hline VRM & 3.1060 & 2.1762 & 1.2629 & 1.0218 \\
\hline RMM & 1.8956 & 1.6118 & 1.9326 & 1.0989 \\
\hline PAP & 3.6894 & 1.7448 & 1.0960 & 1.3611 \\
\hline SPP & 2.1301 & 1.2997 & 1.5675 & 1.7005 \\
\hline NAR & 2.2569 & 1.4510 & 1.7029 & 1.2018 \\
\hline SNR & 1.8826 & 1.2750 & 1.7746 & 1.5675 \\
\hline
\end{tabular}

\subsection{Recognition scores}

Table 3 represents the the percentage of recognition scores for unprocessed and processed signal. Figure 3 represents the graphical comparisons of recognition scores.

\subsection{Recognition grades}

Recognition grades are obtained by grading from 1 to 5.1 represents poor grade and 5 represents Best grades given by the subjects. Figure 4 represents the graphical comparisons of Recognition grades.

Table 4. Comparision of Recognotion Grades

\begin{tabular}{|l|l|l|l|l|}
\hline Subjects & U .S & $\begin{array}{l}\text { P.S } \\
\text { C.bands }\end{array}$ & $\begin{array}{l}\text { P.S } \\
\text { Critical } \\
\text { bands }\end{array}$ & $\begin{array}{l}\text { P.S 1/3 } \\
\text { Octave } \\
\text { bands }\end{array}$ \\
\hline DMM & 3 & 4 & 4 & 5 \\
\hline PPP & 3 & 4 & 4 & 4 \\
\hline APS & 2 & 3 & 5 & 5 \\
\hline PMN & 1 & 3 & 3 & 5 \\
\hline VRM & 4 & 5 & 5 & 5 \\
\hline RMM & 3 & 4 & 5 & 5 \\
\hline PAP & 2 & 3 & 3 & 3 \\
\hline SPP & 2 & 4 & 5 & 5 \\
\hline NAR & 1 & 3 & 4 & 4 \\
\hline SNR & 2 & 3 & 5 & 5 \\
\hline
\end{tabular}

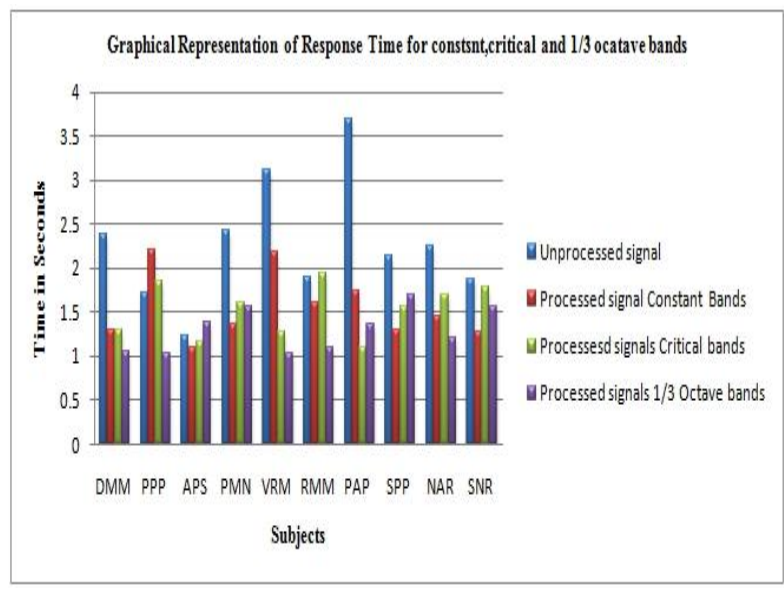

Figure 2: Graphical Comparision of Response Times

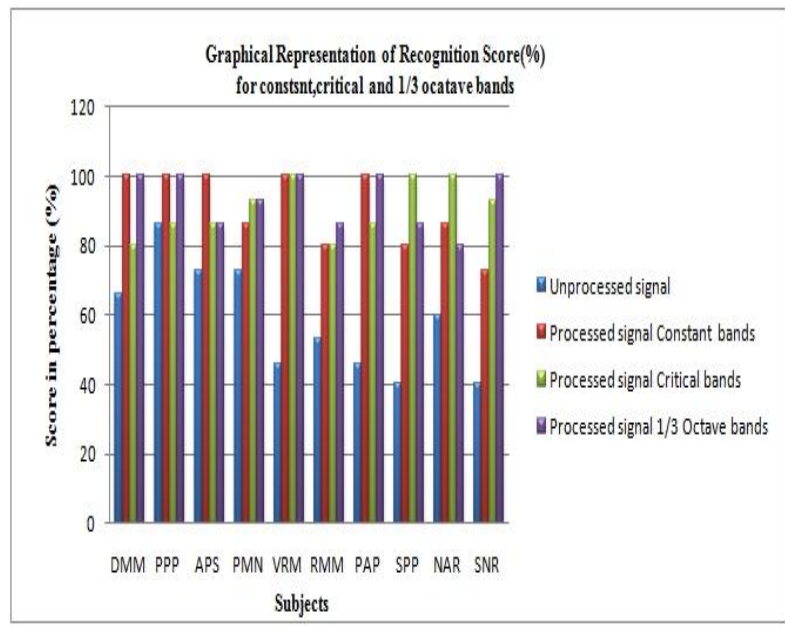

Figure 3: Graphical Comparision of Recognotion Scores 


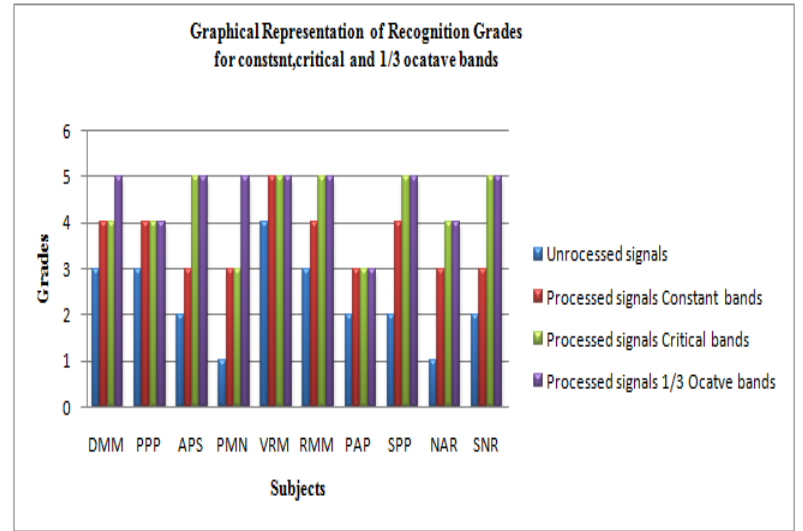

Figure 4: Graphical Comparision of Recognotion Grades

\section{CONCLUSION}

The main advantage of the proposed algorithm is to provide a low cost solution for Sensorineural hearing impairment.From the analysis, it is observed that these schemes gives maximum benefit by reducing the effects of increased masking.In listening tests involving the hearing impaired subjects, the subjects are able to perceptually integrate the dichotically presented speech signal, and the presentation results in improved speech reception. The performed test outcome proves that the projected method is useful for improving the Recognition score, Recognition Grades and also reduces the Response time.

\section{REFERENCES}

[1] Moore, B. C. ), 1997. J,An Introduction to Psychology of Hearing,,4th Ed. (Academic, London.

[2] CHABA,1991.,Speech-perception aids for hearingimpaired people:Current status and needed research,J. Acoust. Soc. Am. vol. 90, 637-683.

[3] GL Ward ,MD MacAllister,1993,Apparatus and method for conveying amplified sound to ear", Google Patent.

[4] Loizou,P.C., Mani, Arunvijay, and Dorman, M.F., 2003,Dichotic speech recognition in noise using reduced spectral cues, J. Acoust. Soc. Am. vol. 114(1), 475-483.
[5] Yasu, K., Kobayashi, K., Hishitani, M., Arai, T and Murahara Y.,2004,Critical band based frequency compression for digital hearing aids, J. Acoust. Sci. and Tech. vol. 25, 61-63.

[6] Deniz Baskent, 2006,Speech recognition in normal hearing and sensorineural hearing loss as a function of the number of spectral channels, J. Acoust. Soc. Am. vol. 120(5), 2908-2925 .

[7] Kulkarni, P.N., and Pandey, P.C.,2008, Optimizing the comb Filters for spectral splitting of speech to reduce the Effect of spectral masking,IEEE-International Conference on Signal processing, Communications and Networking. Madras Institute of Technology, Anna University Chennai India, Jan 4-6, 69-73 .

[8] Daniel M. Rasetshwane, Member, IEEE, Michael P. Gorga, and Stephen T. Neely, Member, IEEE,,2014,Signal-Processing Strategy for Restoration of Cross-Channel Suppression in Hearing-Impaired Listeners",IEEE Transactions On Biomedical Engineering, vol. 61, No. 1, January .

[9] Chanwoo Kim,Kean K.Chin,Michiel Bacchiani,Richard M. Stern,2014,Robust speech recognition using temporal masking and thresholding algorithm, International Science Congress Association ,ISCA

[10] Peng Dai , Frank Rudzicz , Ing Yann Soon , Alex Mihailidis, Huijun Ding ,2015, 2D Psychoacoustic modeling of equivalent masking for automatic speech recognition,ELSEVIER.

[11] Fernando de-la-Calle-Silos, Student Member, IEEE, Francisco J. Valverde Albacete, Member, IEEE,Ascension Gallardo-Antolin, and Carmen Pelaez Moreno, Member, IEEE,2015, Morphologically Filtered Power-Normalized Cochleograms as Robust, Biologically Inspired Features for ASR, IEEETransactions On Audio, Speech, And Language Processing, Vol. 23, No. 11 . 